\title{
Coal-Miner: A Statistical Method for GWA Studies of Quantitative Traits with Complex Evolutionary Origins
}

\author{
Hussein A. Hejase \\ Department of Computer Science and \\ Engineering \\ Michigan State University \\ East Lansing, Michigan 48824 \\ hijazihu@msu.edu
}

\author{
Natalie Vande Pol \\ Department of Plant, Soil and \\ Microbial Sciences \\ Michigan State University \\ East Lansing, Michigan 48824 \\ vandepo7@msu.edu
}

\author{
Gregory M. Bonito \\ Department of Plant, Soil and \\ Microbial Sciences \\ Michigan State University \\ East Lansing, Michigan 48824 \\ bonito@msu.edu
}

\author{
Patrick P. Edger \\ Department of Horticulture \\ Michigan State University \\ East Lansing, Michigan 48824 \\ edgerpat@msu.edu
}

\author{
Kevin J. Liu* \\ Department of Computer Science and \\ Engineering \\ Michigan State University \\ East Lansing, Michigan 48824 \\ kjl@msu.edu
}

\begin{abstract}
Association mapping (AM) methods are used in genome-wide association (GWA) studies to test for statistically significant associations between genotypic and phenotypic data. The genotypic and phenotypic data share common evolutionary origins - namely, the evolutionary history of sampled organisms - introducing covariance which must be distinguished from the covariance due to biological function that is of primary interest in GWA studies. A variety of methods have been introduced to perform AM while accounting for sample relatedness. However, the state of the art predominantly utilizes the simplifying assumption that sample relatedness is effectively fixed across the genome. In contrast, population genetic theory and empirical studies have shown that sample relatedness can vary greatly across different loci within a genome. This phenomenon - referred to as local genealogical variation - is commonly encountered in many genomic datasets. New AM methods are needed to better account for local variation in sample relatedness within genomes.

We address this gap by introducing Coal-Miner, a new statistical AM method. The Coal-Miner algorithm takes the form of a methodological pipeline. The initial stages of Coal-Miner seek to detect candidate loci, or loci which contain putatively associated markers. Subsequent stages of Coal-Miner perform test for association using a linear mixed model with multiple effects which account for sample relatedness locally within candidate loci and globally across the entire genome. Using synthetic and empirical datasets, we compare the statistical power and type I error control of Coal-Miner against state-of-the-art AM methods. The simulation conditions reflect a variety of genomic architectures for complex traits and incorporate
\end{abstract}

${ }^{*}$ To whom correspondence should be addressed.

\section{(c) (i) (-)}

This work is licensed under a Creative Commons

Attribution-NonCommercial-ShareAlike International 4.0 License.

ACM-BCB'17, August 20-23, 2017, Boston, MA, USA.

(c) 2017 Copyright held by the owner/author(s). 978-1-4503-4722-8/17/08.

DOI: http://dx.doi.org/10.1145/3107411.3107490 a range of evolutionary scenarios, each with different evolutionary processes that can generate local genealogical variation. Across the datasets in our study, we find that Coal-Miner consistently offers comparable or typically better statistical power and type I error control compared to the state-of-the-art methods.

\section{CCS CONCEPTS}

-Applied computing $\rightarrow$ Computational genomics; Computational biology; Molecular sequence analysis; Molecular evolution; Computational genomics; Systems biology; Bioinformatics; Population genetics;

\section{KEYWORDS}

genome wide association study; GWAS; population stratification; genealogy; coalescent; Arabidopsis

\section{INTRODUCTION}

Genome-wide association (GWA) studies aim to pinpoint loci with genetic contributions to a phenotype by uncovering significant statistical associations between genomic markers and a phenotypic trait under study. We refer to the computational methods used in a GWA analysis as association mapping (AM) methods. Among the most widely studied organisms in GWA studies are natural human populations and laboratory strains of house mouse. Recently, GWA approaches have been applied to natural populations of other organisms sampled from across the Tree of Life. For example, the 1001 Genomes Consortium study [7] published whole genome sequences for over a thousand samples from globally distributed Arabidopsis populations. In combination with phenotypic data, the genomic sequence data was used in a GWA analysis to pinpoint genomic loci involved in flowering time at two different temperatures. Other recent GWA studies such as the study of Porter et al. [24] have focused on bacteria and other microbes (see [6] for a review of relevant literature).

Regardless of sampling strategy - from one or more closely related populations involving a single species to multiple populations from divergent species - it is well understood that sample relatedness can be a confounding factor in GWA analyses unless 
properly accounted for. Intuitively, the genotypes and phenotypes of present-day samples reflect their shared evolutionary history, or phylogeny. For this reason, covariance due to a functional relationship between genotypic markers and a phenotypic character must be distinguished from shared covariance due to common evolutionary origins. A number of AM methods have been developed to address this issue. EIGENSTRAT [26] is a popular AM method which accounts for sample relatedness as a fixed effect. Other statistical AM methods have utilized linear mixed models (LMMs) to capture sample relatedness using random effects; these include EMMAX [17] and GEMMA [31]. Local variation in functional covariance across the genome is a crucial signature that AM methods use to uncover putatively associated markers. In contrast, virtually all of the most widely used state-of-the-art AM methods assume that covariance due to sample relatedness does not vary appreciably across the genome. Sample relatedness is therefore evaluated "globally" across the genome, eliding over "local" genealogical variation across loci. The latter has been observed by many comparative genomic and phylogenomic studies (see [10] for a review of relevant literature). It is now well understood that local genealogical variation within genomes is pervasive across a range of evolutionary divergence - from structured populations within a single species to multiple species at various scales up to the Tree of Life, the evolutionary history of all living organisms on Earth. The evolutionary processes that can contribute to local genealogical variation include genetic drift and incomplete lineage sorting, recombination, gene flow, positive selection, and the combination of all of these processes (and others) [10].

Computational approaches for detecting local genealogical variation are broadly characterized by their modeling assumptions. One class of methods makes use of the Four-Gamete Test [14], which requires the simplifying assumption that sequence evolution can be described by the infinite sites model. The LRScan algorithm [29] belongs to this class of methods. Another class consists of parametric methods that make use of finite-sites models of sequence evolution. These include methods such as RecHMM [30]. More recently, coalescent-based methods such as PhyloNet-HMM [19] have been developed to infer local coalescent histories and explicitly ascribe local genealogical variation to different evolutionary processes.

Building upon these insights, we previously developed Coal-Map [12], an AM method that utilizes a fixed effects model to account for global sample relatedness and, depending upon whether the test marker is located within a locus containing putatively associated markers, local sample relatedness as well. The latter condition is evaluated using model selection criteria. Coal-Map requires local-phylogeny-switching breakpoints as input. We conducted a simulation study which demonstrated that Coal-Map's statistical power and type I error control was comparable or better than other state-of-the-art methods that account for global sample relatedness using fixed effects.

\section{METHODS}

\subsection{Overview of Coal-Miner algorithm}

In this study, we introduce Coal-Miner, a new statistical AM method which accounts for local variation of sample relatedness across genomic sequences as well as global sample relatedness. CoalMiner's contributions relative to the state of the art (including CoalMap) consist of the following. First, Coal-Miner utilizes an LMM with multiple effects to explicitly capture the genomic architecture of a phenotype, where both genotypic and phenotypic characters are the product of a complex evolutionary history which can cause sample relatedness to vary locally across genomic loci. The LMM captures global sample relatedness as a random effect, in contrast to the fixed-effect approach used by Coal-Map. Second, the pipelinebased design of Coal-Miner incorporates an intermediate stage to infer "candidate loci" for use in the new LMM, where a candidate locus is a locus that is inferred to contain one or more putatively associated SNPs.

We begin by introducing the high-level design of Coal-Miner. The input to the Coal-Miner algorithm consists of: (1) an $n \times k$ multilocus sequence data matrix $X$, (2) an $n \times 1$ vector $\boldsymbol{y}$ which represents a phenotypic character, and (3) $\ell^{*}$, the number of candidate loci used during analysis. The output consists of an association score for each site $x \in X$.

Coal-Miner's statistical model captures the relationship between genotypic data $X$ and the phenotypic character $\boldsymbol{y}$ in the form of a linear mixed model (LMM). The LMM incorporates multiple effects to capture the phenotypic contributions of and local genealogical variation among multiple candidate loci. A candidate locus is represented by a fixed effect, and a random effect is included to capture global sample relatedness as measured across all loci in $X$. Ideally, the set of candidate loci identified during a Coal-Miner analysis is identical to the set of causal loci (i.e., loci containing causal SNPs) for the trait under study; in practice, the set of candidate loci are inferred as part of the Coal-Miner algorithm, which we discuss in greater detail below. The LMM takes the following form (based on the notation of Zhou and Stephens [31]):

$$
\begin{aligned}
& \boldsymbol{y}=\boldsymbol{W} \boldsymbol{\alpha}+\boldsymbol{x} \beta+\boldsymbol{u}+\boldsymbol{\epsilon} \\
& \boldsymbol{u} \sim \mathrm{MVN}_{n}\left(0, \lambda \tau^{-1} \boldsymbol{K}_{\text {global }}\right) \\
& \boldsymbol{\epsilon} \sim \operatorname{MVN}_{n}\left(0, \tau^{-1} \boldsymbol{I}_{\boldsymbol{n}}\right)
\end{aligned}
$$

The fixed effects are represented by $c$ covariates in the $n \times c$ matrix $W$, which include covariates that capture local sample relatedness within each candidate locus, the $c \times 1$ vector $\boldsymbol{\alpha}$ of corresponding coefficients, and the test SNP is represented by the $n \times 1$ vector $\boldsymbol{x}$ with effect size $\beta$. Global sample relatedness (i.e., sample relatedness as measured across all loci in the genotypic data $X$ ) is specified by the $n \times n$ relatedness matrix $\boldsymbol{K}_{\text {global }}$ computed using $X$, following the approach of state-of-the-art LMM-based AM methods (e.g., GEMMA [31]). The $n \times 1$ vectors $\boldsymbol{u}$ and $\boldsymbol{\epsilon}$ represent random effects which account for global sample relatedness and residual error, respectively. Each of the two random effects follows an $n$ dimensional multivariate normal distribution (abbreviated "MVN") with mean 0 . The random effects $\boldsymbol{u}$ have covariance $\lambda \tau^{-1} K_{\text {global }}$ and the random effects $\boldsymbol{\epsilon}$ have covariance $\tau^{-1} \boldsymbol{I}_{\boldsymbol{n}}$, where $\lambda$ is the relative ratio between the two, $I_{\boldsymbol{n}}$ is the $n \times n$ identity matrix, and the residual errors have variance $\tau^{-1}$.

The design of the Coal-Miner algorithm takes the form of a methodological pipeline. We now discuss each pipeline stage in turn. 
Stage one of Coal-Miner: inferring local-phylogeny-switching breakpoints. The input to the first stage of Coal-Miner is the genotypic data matrix $X$. The output consists of a set of local-phylogenyswitching breakpoints $\boldsymbol{b}$ which partition the sites in $X$ into loci $\left\{X_{i}\right\}$, where $1 \leq i \leq \ell$ and $\ell$ is the number of loci. We require that $\ell^{*} \leq \ell$. (The ratio of $\ell^{*}$ and $\ell$ depends upon the genomic architecture of the trait corresponding to character $\boldsymbol{y}$.)

The general approach to address this computational problem is to infer local coalescent histories under an appropriate multi-species extension of the coalescent model [18], and then to assign breakpoints based upon gene tree discordance. Each pair of neighboring breakpoints delineates a locus for use in downstream stages of the Coal-Miner pipeline. The specific choice of model/method depends upon the relevant evolutionary processes involved in multi-locus sequence evolution, particularly regarding the source(s) of local genealogical discordance.

In this study, we use one of two different methods, depending upon assumptions about biomolecular sequence evolution. In the simulation study, the simulations make use of the infinite sites model. We therefore used the LRScan algorithm [29] to compute local-topology-switching breakpoints based upon the Four Gamete Test (FGT) [14]. In the empirical study, we did not make use of the infinite sites model and its assumptions about sequence evolution. Furthermore, multiple evolutionary processes were known to be involved in multi-locus sequence evolution, including genetic drift/incomplete lineage sorting (ILS), recombination/gene conversion, gene flow/horizontal gene transfer (HGT), and natural selection. Breakpoint inference under the corresponding extended coalescent model is suspected to be a computationally difficult problem. Existing methods for this problem (e.g., PhyloNet-HMM [19]) did not have sufficient scalability for the dataset sizes examined in our study. As a more feasible alternative, we inferred local-topologyswitching breakpoints using Rec-HMM [30]. Rec-HMM performs fixed-species-phylogeny inference of local genealogies under a statistical model that combines a finite-sites substitution model and a hidden Markov model which is meant to capture intra-sequence dependence (such as arises from recombination).

Stage two of Coal-Miner: identifying candidate loci. The input to the second stage of Coal-Miner consists of the genotypic data matrix $X$, the set of breakpoints $\boldsymbol{b}$ which partition $X$ into loci $\left\{X_{i}\right\}$, where $1 \leq i \leq \ell$ and $\ell$ is the number of loci, the phenotypic character $\boldsymbol{y}$, and $\ell^{*}$, the number of candidate loci to identify. Note that the input $\boldsymbol{b}$ is an output of the preceding stage of Coal-Miner. The output is a set of candidate loci $\left\{X_{j}^{*}\right\} \subseteq\left\{X_{i}\right\}$ where $1 \leq j \leq \ell^{*}$.

Our general approach to this problem consists of a search among possible sets of candidate loci $\left\{X_{j}^{*}\right\}$ using optimization under a "null" version of Coal-Miner's LMM, where we do not consider a test SNP (i.e., $\beta=0$ in Coal-Miner's LMM) and the phenotypic contributions from putatively associated SNPs in each candidate locus $X_{j}^{*}$ is captured by covariates $\left\{\boldsymbol{w}_{j}\right\} \subseteq W$. Since we compare fitted LMMs that may have varying fixed effects, our optimization criterion consists of the LMM $\log$-likelihood $\mathcal{L}(\lambda, \tau, \boldsymbol{\alpha}, \beta)=\frac{n}{2} \log (\tau)-$ $\frac{n}{2} \log (2 \pi)-\frac{1}{2} \log |\boldsymbol{H}|-\frac{1}{2} \tau(\boldsymbol{y}-\boldsymbol{W} \boldsymbol{\alpha}-\boldsymbol{x} \beta)^{T} \boldsymbol{H}^{-1}(\boldsymbol{y}-\boldsymbol{W} \boldsymbol{\alpha}-\boldsymbol{x} \beta)$ where $\boldsymbol{H}=\lambda \boldsymbol{K}_{\text {global }}+\boldsymbol{I}_{n}$ (reproduced from equation (3) in [31]). Due to the computational difficulty of this optimization problem, numerical optimization procedures are typically used. We obtained estimates of $\lambda$ in the range of $\left[10^{-5}, 1\right]$ using the optimization heuristic implemented in the GEMMA software library [31], which combines Brent's method [5] and the Newton-Raphson method.

For each candidate locus $X_{j}^{*}$, local sample relatedness was evaluated using principal component analysis (PCA) [16] of $X_{j}^{*}$ - similar to techniques that are widely used by AM methods to account for global sample relatedness as fixed effects [26]. The phenotypic contribution of candidate locus $X_{j}^{*}$ was represented using covariates $\left\{\boldsymbol{w}_{j}\right\}$ which consisted of the top five principal components, where the $z$ th principal component corresponds to the sample covariance matrix eigenvector with the $z$ th largest eigenvalue and the number of covariates was based upon a design experiment in [12]. For added computational efficiency, we substituted the following search heuristic in place of set-based search among all possible $\ell^{*}$-size sets of candidate loci. For each locus $X_{i}$, we used MLE to fit an equivalent LMM, except that the covariates $W$ included only the covariates $\left\{\boldsymbol{w}_{\boldsymbol{i}}\right\}$ for locus $\boldsymbol{X}_{\boldsymbol{i}}$ (as computed using the above PCAbased procedure). The output set of candidate loci consists of the top $\ell^{*}$ loci based upon fitted LMM likelihood.

Stage three of Coal-Miner: SNP-based association testing. The input to the third stage of Coal-Miner consists of the genotypic data matrix $X$, the set of breakpoints $\boldsymbol{b}$ which partition $X$ into loci $\left\{X_{i}\right\}$, where $1 \leq i \leq \ell$ and $\ell$ is the number of loci, the phenotypic character $\boldsymbol{y}$, and the set of candidate loci $\left\{X_{j}^{*}\right\}$. Note that the inputs $\boldsymbol{b}$ and $\left\{X_{j}^{*}\right\}$ are outputs of stages one and two of Coal-Miner, respectively. The output of this stage is Coal-Miner's final output.

Each test SNP $\boldsymbol{x}$ is tested for association under Coal-Miner's LMM. Variation in local sample relatedness across candidate loci $\left\{X_{j}^{*}\right\}$ is captured by covariates in $W$ : specifically, if the test SNP $x$ is located within a candidate locus $X_{j}^{*}$, the covariates $W$ include a corresponding covariate $\boldsymbol{w}_{j}$ which consists of the top principal component from PCA applied to $X_{j}^{*}$ (see above discussion of previous stage), and otherwise not. (Stages two and three of the Coal-Miner pipeline utilize different covariates $W$ due to the absence or presence of a test SNP effect in their respective LMMs.) The LMM is fitted using the likelihood-based numerical optimization procedures that are also used in stage two of Coal-Miner, and the association score is computed using a likelihood ratio test of the fitted model against a null model with no SNP effect.

\subsection{Simulation study}

Neutral simulations of multi-locus sequence data were based upon either tree-like or non-tree-like evolutionary scenarios. The evolutionary scenarios shared a species phylogeny that we used in a prior simulation study (Supplementary Figure S1 in the Supporting Online Materials (SOM)). We used ms [13] to simulate coalescent histories (and embedded gene trees) under an extension of the coalescent model [18] which allows instantaneous unidirectional admixture (IUA) [9]. Under this model, the parameterization of the model phylogeny includes an admixture proportion $\gamma$. Appropriate choices of $y$ allow us to explore the impact of tree-like and non-tree-like evolution in our simulation study, where we set $\gamma$ to either 0.0 or 0.5 , respectively. Each replicate dataset sampled 10 independently and identically distributed loci and 1000 individuals; taxa A, B, and C were represented by 250,250 , and 500 samples, 
respectively. Bi-allelic sequence evolution was simulated under the infinite sites model to obtain $250 \mathrm{bp}$ per locus, resulting in total sequence length of $2.5 \mathrm{~kb}$ per replicate dataset.

As a means to investigate the impact of the genomic architecture of phenotypes, we simulated phenotypic characters using the approach from our previous work [12]. For each synthetic multi-locus sequence dataset in the neutral simulations, we randomly selected either $10 \%, 20 \%$, or $30 \%$ of loci as causal. Twenty causal SNPs were then randomly selected from causal loci such that each causal locus contained at least one causal SNP and causal SNPs had minor allele frequency between 0.1 and 0.3. Given a set of causal SNPs $\delta$, we sampled character $y$ under an extension of the quantitative trait model used by Long and Langley [20]. The trait value for the $i$ th individual is represented as $y_{i}=\pi \sum_{j \in \delta} \frac{Q_{i, j}}{|\delta|}+(1-\pi) N(0,0.01)$ where $\pi$ specifies the ratio between the genotypic contribution and an environmental residual, $Q$ is 1 if sample $i$ has the derived allele at the $j$ th causal SNP and 0 otherwise, and the environmental residual is normally distributed with mean 0 and standard deviation 0.01 . Our simulations utilized a ratio $\pi$ of 0.5 .

Our simulation study also included non-neutral simulations that incorporated positive selection. We used msms [11] to conduct forward-time coalescent simulations of genotypic sequence evolution (in place of an otherwise equivalent neutral backward-time coalescent simulation using ms), where causal loci were evolved under deme-dependent positive selection with a finite sites mutation model and all other loci evolved neutrally (as discussed above in the neutral simulation procedure). We used a selection coefficient of $s=0.56$, which is in line with estimates from prior studies of positive selection in natural Mus populations [28]. Quantitative traits were simulated using the above procedure.

The simulation study experiments involving quantitative traits with varying genomic architectures included 12 different model conditions in total. To recap, the model conditions differed in terms of the proportion of causal loci (either $10 \%, 20 \%$, or $30 \%$ ), model phylogeny (either tree-like or non-tree-like), and the presence or absence of positive selection. For each model condition, we repeated the simulation procedure to obtain 20 replicate datasets.

Performance evaluation. The other methods in our study consisted of Coal-Map, GEMMA, and EIGENSTRAT. We followed the procedure from [12] to obtain FGT-based local-phylogenyswitching breakpoints and run Coal-Map analyses. For consistency with the other LMM-based AM methods in our study, we ran GEMMA using an IBS kinship matrix as our measure of global sample relatedness and MLE and LRT to obtain association scores. EIGENSTRAT was run with default settings using the top ten principal components from the genotypic data matrix $X$, following the recommendations of Price et al. [26]. Detailed software commands are listed in the SOM Appendix.

We evaluated performance based on statistical power, type I error, and AUROC. To compare AUROC, we performed DeLong et al. tests [8] using the Daim v. 1.1.0 package [25] in $\mathrm{R}$ [27]. Custom scripts were used to conduct the simulation study. All scripts are provided under an open source license. See SOM Appendix for details and download instructions.

\subsection{Empirical study}

We re-analyzed an Arabidopsis dataset which consists of whole genome sequence (WGS) data and phenotypic data for two quantitative traits: flowering time at $10^{\circ} \mathrm{C}$ and $16^{\circ} \mathrm{C}$. A total of 1,135 samples from natural populations across the globe are represented. The phylogeny shown in SOM Supplementary Figure S16 depicts the geographic origins of and evolutionary relationships among the samples. The dataset was originally published and analyzed by the 1001 Genomes Consortium [7], and we obtained genomic sequences and quantitative trait data from the 1001 Genomes Project database (accessible at www.1001genomes.org); the former includes both assembled WGS data and variant calls for a total of 10,707,430 biallelic SNPs. (Details about sequencing, assembly, filtering, quality controls, and variant calling are described in the 1001 Genomes Consortium study [7].)

Stage one of the Coal-Miner pipeline made use of RecHMM [30] to infer local-phylogeny-switching breakpoints. For computational efficiency, the breakpoint inference utilized a subset of taxa rather than the full set of taxa. The subset was chosen to maximize evolutionary divergence and was comprised of one sample from each of the following geographic regions: Spain, Sweden, USA, and Russia. For chromosomes 1 through 5, the analysis in stage one resulted in 1876, 991, 783, 559, and 913 loci with an average locus length of 16 kb, $19 \mathrm{~kb}, 30 \mathrm{~kb}, 33 \mathrm{~kb}$, and $29 \mathrm{~kb}$, respectively.

Using the loci obtained in stage one as input, the second stage of Coal-Miner was run on both trait characters. The $10^{\circ} \mathrm{C}$ analysis identified 179, 99, 108, 109, and 95 candidate loci in chromosomes 1 through 5 , respectively. The $16^{\circ} \mathrm{C}$ analysis identified $115,42,88$, 65 , and 89 candidate loci in chromosomes 1 through 5 , respectively. Coal-Miner also requires that $\ell^{*}$, the number of candidate loci, be provided as an input parameter. In practice, model selection approaches are typically used in this context. Our study utilized the following procedure to determine a suitable value for $\ell^{*}$. We calculated the likelihood score of the fitted "null" LMM for each locus (see above), and we examined the distribution of likelihood scores (Supplementary Figure S15 in the SOM). We then assigned $\ell^{*}$ based on the distribution's inflection point.

The inputs to the third stage of Coal-Miner consisted of the set of candidate loci, a quantitative trait character (flowering time at either $10^{\circ} \mathrm{C}$ or $16^{\circ} \mathrm{C}$ ), and the genotypic sequence data matrix which consisted of sites with minor allele frequency threshold of 0.03 (i.e., sites having a minor allele frequency less than or equals to 0.03 were removed). The third stage of Coal-Miner was run using the same settings as in the simulation study.

\section{RESULTS}

\subsection{Simulation study}

We conducted experiments that varied the proportion of causal loci as a means to investigate the impact of the genomic architecture of a trait on AM method performance. The model conditions utilized simulations with between $10 \%$ and $30 \%$ causal loci and either neutral or non-neutral evolution on either tree-like or non-tree-like model phylogenies. The methods under study included Coal-Miner, our new AM method, as well as representative methods from different classes of state-of-the-art methods: Coal-Map, an AM method that accounts for local and global sample relatedness as fixed effects, 
GEMMA, a LMM-based AM method that accounts for global sample relatedness as a random effect (but does not account for local sample relatedness), and EIGENSTRAT, an AM method that accounts for global sample relatedness as a fixed effect (but does not account for local sample relatedness). We compared the statistical power and type I error control of each method using receiver operating characteristic (ROC) curves (Supplementary figures S2 through $\mathrm{S} 5$ in the SOM), and Table 1 compares the area under ROC curve (AUROC) of each method.

Regardless of the proportion of causal loci and the evolutionary scenario explored in these model conditions, Coal-Miner's AUROC was significantly better than the next best method in our study (either Coal-Map or GEMMA) based upon the corrected test of DeLong et al. [8] (Table 1). A similar observation was made when measuring performance using true positive rate (TPR) at a false positive rate (FPR) of 0.1 (Supplementary Table S2 in the SOM), except that Coal-Miner's performance advantage over the next best method was even more pronounced. The TPR difference was 0.158 on average and ranged as high as 0.248 . Across these model conditions, we observed a consistent ranking of AM methods by AUROC (with two minor exceptions): Coal-Miner first, Coal-Map second, GEMMA third, and EIGENSTRAT fourth. The minor exceptions involved the two lowest AUROC values on the neutral, non-treelike model condition with $10 \%$ or $20 \%$ causal loci, where GEMMA and EIGENSTRAT swapped rankings. We noted that Coal-Map's AUROC was second best on model conditions with the smallest proportion of causal loci, but its performance tended to degrade as the proportion increased. Coal-Map's AUROC was only marginally better than GEMMA on model conditions with the highest proportion of causal loci.

The impact of varying the proportion of causal loci was similar for all methods: AUROC tended to degrade as the proportion of causal loci increased from $10 \%$ to $30 \%$. However, Coal-Miner's performance advantage relative to the other AM methods was flat or improved as the proportion of causal loci increased.

The model conditions included different combinations of genetic drift/incomplete lineage sorting and/or gene flow - evolutionary processes which can generate local variation in sample relatedness. Note that model conditions with non-tree-like model phylogenies incorporated all of these evolutionary processes (including genetic drift/incomplete lineage sorting). The impact of the different evolutionary processes varied across the methods. Coal-Miner's AUROC tended to be larger on model conditions involving both drift/ILS and gene flow as sources of local genealogical variation, and CoalMap's AUROC was similarly affected. On the other hand, GEMMA's AUROC was comparable (within 0.01) based on this comparison, with the exception of non-neutral model conditions involving $10 \%$ or $20 \%$ causal loci.

A comparison of model conditions that differed only with respect to neutral versus non-neutral evolution revealed the impact of positive selection on AM method performance. We note that, in our experiments, the evolution of causal loci differed from non-causal loci since positive selection acted only upon the former but not the latter. Coal-Miner and Coal-Map returned comparable AUROC (within 0.025) regardless of neutral versus non-neutral evolution. GEMMA and EIGENSTRAT performed similarly, although slightly greater variability (within 0.035 ) was observed. For LMM-based methods, there was no obvious trend in terms of direction of change when comparing neutral versus non-neutral experiment results. There was an apparent trend for EIGENSTRAT, however: positive selection tended to reduce EIGENSTRAT's AUROC, with one exception (model conditions with a tree-like model phylogeny and $10 \%$ causal loci).

\subsection{Empirical study}

We used Coal-Miner to re-analyze an Arabidopsis dataset which was originally studied by the 1001 Genomes Consortium [7]. The dataset includes samples from 1,135 high quality re-sequenced natural lines adapted to different environments with varying local climates [7]. The sampled data included whole genome sequences and quantitative trait data for two traits: flowering time under high and low temperature $-16^{\circ} \mathrm{C}$ and $10{ }^{\circ} \mathrm{C}$, respectively.

A key component of the 1001 Genomes Consortium study was a GWA analysis of the genomic sequences and quantitative trait data using EMMAX [17], another state-of-the-art statistical AM method (see [31] for a comparison of EMMAX and other stateof-the-art statistical AM methods). A major focus of the analysis was a set of five genes which are known to regulate flowering and contribute to flowering time variation at $10^{\circ} \mathrm{C}$ in Arabidopsis [7]: FLOWERING LOCUS T (FT), SHORT VEGETATIVE PHASE (SVP), FLOWERING LOCUS C (FLC), DELAY OF GERMINATION 1 (DOG1), and VERNALIZATION INSENSITIVE 3 (VIN3). Plants rely on both endogenous and environmental (e.g. temperature and photoperiod) cues to initiate flowering $[1,2]$. These five genes encode major components of the vernalization (exposure to the prolonged cold) and autonomous pathways known to regulate the initiation of flowering in Arabidopsis. Allelic and copy number variants $(\mathrm{CNV})$ for many of these genes, including FLC, are known to serve important roles in generating novel variation in flowering time and permit plants to adapt to new climates [21-23]. DOG1 is known to be involved in determining seasonal timing of seed germination and influences flowering time in Arabidopsis [15].

Under a conservative Bonferroni-corrected threshold [4], CoalMiner identified significant peaks associated with flowering time under high and low temperature $\left(16^{\circ} \mathrm{C}\right.$ and $10^{\circ} \mathrm{C}$, respectively). In particular, Coal-Miner identified significantly associated markers in all five genes (FT, SVP, FLC, DOG1, and VIN3) for both the $16^{\circ} \mathrm{C}$ dataset and the $10^{\circ} \mathrm{C}$ dataset (Supplementary Figure S12 in the SOM). Within the five genes, Coal-Miner analyses returned peaks which largely agreed across the $10{ }^{\circ} \mathrm{C}$ and $16^{\circ} \mathrm{C}$ datasets. Some differences involved association scores that were borderline significant in one dataset but not the other.

Table 2 compares the Coal-Miner analysis with similar analyses using two other state-of-the-art statistical AM methods. The EMMAX analysis in the 1001 Genomes Consortium study [7] identified significant associations for three of the genes at $10{ }^{\circ} \mathrm{C}$, and association score peaks were marginally below a Bonferroni-corrected threshold in the other two genes (SVP and FLC). Furthermore, significant peaks were only detected in DOG1 at $16^{\circ} \mathrm{C}$, but no significant peaks were detected in the other four genes for this dataset. (See Figure 2 in the 1001 Genomes Consortium study [7] for the original Manhattan plot.) GEMMA's performance was qualitatively similar to EMMAX (Supplementary Figure S13 in the SOM). At $10^{\circ} \mathrm{C}$, 


\begin{tabular}{|c|c|c|c|c|c|c|c|}
\hline \multicolumn{3}{|c|}{ Model condition } & \multicolumn{4}{|c|}{ AUROC } & \multirow[b]{2}{*}{ q-value } \\
\hline $\begin{array}{l}\text { Neutral vs. } \\
\text { non-neutral }\end{array}$ & $\begin{array}{c}\text { Model } \\
\text { phylogeny }\end{array}$ & $\begin{array}{l}\text { Percentage of } \\
\text { causal loci }(\%)\end{array}$ & Coal-Miner & Coal-Map & GEMMA & EIGENSTRAT & \\
\hline \multirow[t]{3}{*}{ Neutral } & \multirow[t]{3}{*}{ Non-tree-like } & 10 & $0.962(0.009)$ & $0.939(0.009)$ & $0.866(0.017)$ & $0.871(0.014)$ & $<0.00001$ \\
\hline & & 20 & $0.921(0.010)$ & $0.899(0.009)$ & $0.849(0.015)$ & $0.859(0.012)$ & $<0.00001$ \\
\hline & & 30 & $0.904(0.013)$ & $0.882(0.009)$ & $0.847(0.017)$ & $0.832(0.018)$ & $<0.00001$ \\
\hline \multirow[t]{3}{*}{ Neutral } & \multirow[t]{3}{*}{ Tree-like } & 10 & $0.943(0.014)$ & $0.922(0.010)$ & $0.870(0.009)$ & $0.833(0.019)$ & 0.0053 \\
\hline & & 20 & $0.904(0.016)$ & $0.847(0.011)$ & $0.843(0.010)$ & $0.813(0.016)$ & $<0.00001$ \\
\hline & & 30 & $0.904(0.013)$ & $0.853(0.009)$ & $0.844(0.008)$ & $0.799(0.022)$ & 0.00003 \\
\hline \multirow[t]{3}{*}{ Non-neutral } & \multirow[t]{3}{*}{ Non-tree-like } & 10 & $0.959(0.009)$ & $0.933(0.013)$ & $0.896(0.014)$ & $0.836(0.022)$ & $<0.00001$ \\
\hline & & 20 & $0.926(0.009)$ & $0.897(0.009)$ & $0.856(0.017)$ & $0.847(0.013)$ & $<0.00001$ \\
\hline & & 30 & $0.894(0.015)$ & $0.863(0.010)$ & $0.832(0.018)$ & $0.816(0.014)$ & $<0.00001$ \\
\hline \multirow[t]{3}{*}{ Non-neutral } & \multirow[t]{3}{*}{ Tree-like } & 10 & $0.954(0.014)$ & $0.922(0.010)$ & $0.856(0.012)$ & $0.841(0.018)$ & $<0.00001$ \\
\hline & & 20 & $0.890(0.015)$ & $0.850(0.013)$ & $0.832(0.014)$ & $0.796(0.020)$ & 0.00003 \\
\hline & & 30 & $0.879(0.014)$ & $0.836(0.011)$ & $0.830(0.009)$ & $0.783(0.018)$ & 0.0007 \\
\hline
\end{tabular}

Table 1: The impact of the genomic architecture of a quantitative trait on the performance of Coal-Miner and the other AM methods. Multi-locus sequences were simulated under neutral or non-neutral evolution on tree-like or non-tree-like model phylogenies, and quantitative traits were simulated using causal markers sampled from $10 \%, 20 \%$, or $30 \%$ of loci (see Methods section for more details). The performance of each AM method was evaluated based on the area under its receiver operating characteristic (ROC) curve, or AUROC. We report each method's AUROC as an average (and standard error in parentheses) across twenty replicate datasets for each model condition. Coal-Miner's AUROC is shown in bold where it significantly improved upon the AUROC of the most accurate of the other AM methods, based upon the test of DeLong et al. [8] $(n=20$; $\alpha=0.05)$. We corrected for multiple tests using the approach of Benjamini and Hochberg [3], and corrected q-values are shown. (The corresponding ROC plots are shown in Supplementary Figures S2 through S5 in the SOM.)

GEMMA recovered significant associations in three of the genes but not in the remaining two (SVP and FLC); at $16^{\circ} \mathrm{C}$, no significant peaks were detected in three genes, a peak just above the threshold of significance was detected in FT, and another peak was detected in DOG1.

\section{DISCUSSION}

Simulation study. For the model conditions that varied the proportion of causal loci with neutral or non-neutral evolution on tree-like or non-tree-like model phylogenies, Coal-Miner had better performance than all of the other state-of-the-art methods in our study, as measured using AUROC and TPR at an FPR of 0.1. This suggests that Coal-Miner's performance advantage is robust to the specific proportion of causal loci that contribute genetic effects to a quantitative trait, which relates to trait architecture, as well as the evolutionary processes involved. We note that, as even more causal loci are added beyond the proportions explored in our study, the effects contributed by any individual locus becomes more diffuse, and global sample structure will become a more reasonable approximation of different causal loci with different local sample structures. In general, we found traits with "diffuse" genomic architecture (i.e., traits with a relatively high proportion of causal loci) to be challenging for all methods. Coal-Miner tended to cope better with the challenge relative to the other methods in our study, which we attribute to the design of the second stage in the Coal-Miner pipeline (i.e., candidate locus detection). Consistent performance trends were observed when comparing neutral versus non-neutral simulations. This suggests that, for the model conditions that we explored in our study, Coal-Miner's performance is robust to the presence or absence of positive selection. A similar outcome was observed when comparing IUA model-based experiments involving two different types of model phylogenies - tree-like and non-treelike.

Taken together, the model conditions included multiple sources of local genealogical variation, including genetic drift/ILS, gene flow, positive selection, and combinations thereof. The specific evolutionary processes contributing to local genealogical variation did not seem to matter as much as the presence of local genealogical variation, and Coal-Miner's performance advantage was not necessarily predicated on specific evolutionary cause(s) of local genealogical discordance. These findings seem to suggest that Coal-Miner's model and algorithm may be generalized to other evolutionary scenarios, so long as the breakpoint inference method used in the Coal-Miner pipeline suitably accounts for evolutionary processes with first-order contributions to genome evolution. An additional consideration is that the simulations utilized minor allele frequencies of at least 0.1 , and future work is needed to understand Coal-Miner's performance in GWA studies involving rare variants.

Empirical study. The empirical datasets in our study were more challenging than the simulated datasets because the former likely involved more complex evolutionary evolutionary scenarios compared to the latter. Additional evolutionary processes which may have played an important role include other types of natural selection and demographic events (e.g., fluctuations in effective population size).

For both of the Arabidopsis datasets, Coal-Miner was able to detect significant associations in all five positive control regions. In contrast, neither GEMMA nor EMMAX - the statistical AM method used in the 1001 Genomes Consortium study [7] - were able to do the same. The vernalization requirement for flowering in 


\begin{tabular}{|c|c|c|c|c|}
\hline & & \multicolumn{3}{|c|}{ Significantly associated markers detected? } \\
Dataset & Positive control gene & Coal-Miner & EMMAX & GEMMA \\
\hline $10^{\circ} \mathrm{C}$ & FLOWERING LOCUS T (FT) & Yes & Yes & Yes \\
$10^{\circ} \mathrm{C}$ & SHORT VEGETATIVE PHASE (SVP) & Yes & No* & No \\
$10^{\circ} \mathrm{C}$ & FLOWERING LOCUS C (FLC) & Yes & No* $^{*}$ & No \\
$10^{\circ} \mathrm{C}$ & DELAY OF GERMINATION 1 (DOG1) & Yes & Yes & Yes \\
$10^{\circ} \mathrm{C}$ & VERNALIZATION INSENSITIVE 3 (VIN3) & Yes & Yes & Yes \\
\hline $16^{\circ} \mathrm{C}$ & FLOWERING LOCUS T (FT) & Yes & No & Yes \\
$16^{\circ} \mathrm{C}$ & SHORT VEGETATIVE PHASE (SVP) & Yes & No & No \\
$16^{\circ} \mathrm{C}$ & FLOWERING LOCUS C (FLC) & Yes & No & No \\
$16^{\circ} \mathrm{C}$ & DELAY OF GERMINATION 1 (DOG1) & Yes & Yes & Yes \\
$16^{\circ} \mathrm{C}$ & VERNALIZATION INSENSITIVE 3 (VIN3) & Yes & No & No \\
\hline
\end{tabular}

Table 2: A comparison of Coal-Miner and two other state-of-the-art statistical AM methods based upon analyses of the two Arabidopsis datasets. The other AM methods are GEMMA and EMMAX, the statistical AM method used in the 1001 Genomes Consortium study [7]. We evaluated whether the three AM methods detected significantly associated markers in five genomic regions centered on positive control genes which are known to regulate flowering time in Arabidopsis. We used a Bonferronicorrected threshold for significance. For two of the five genomic regions in the $10^{\circ} \mathrm{C}$ dataset, EMMAX returned association scores that were near the threshold of significance (marked using an asterisk). The corresponding Manhattan plots for the CoalMiner and GEMMA analyses are shown in Supplementary Figures S12 and S13 in the SOM, respectively. The corresponding Manhattan plot for the EMMAX analysis is shown as Figure 2 in the 1001 Genomes Consortium study [7].

Arabidopsis suggests that the flowering response at $16{ }^{\circ} \mathrm{C}$ presents a greater $\mathrm{AM}$ challenge than at $10^{\circ} \mathrm{C}$. Our findings were consistent with a need for more statistical power for the former as compared with the latter as well as the overall findings in the simulation study, which suggested that Coal-Miner offered improved statistical power relative to the state of the art. As noted above, the empirical datasets likely involved relatively complex evolutionary histories as compared to the synthetic datasets in our study, and an expanded simulation study would be needed to confirm our initial comparison of performance findings using synthetic and empirical data. Furthermore, Coal-Miner analysis of the Arabidopsis dataset identified putatively novel markers (i.e., markers which were not flagged using other AM methods). Additional comparative and functional analyses are needed to interpret these findings.

\section{CONCLUSIONS}

Across the range of genomic architectures and evolutionary scenarios explored in our study, Coal-Miner had comparable or typically improved statistical power and type I error control compared to state-of-the-art AM methods. The scenarios included different evolutionary processes such as genetic drift and ILS, positive selection, gene flow, and recombination - all of which can generate local genealogical variation that differs from the true species phylogeny. More work needs to be done to explore additional evolutionary processes which have first-order impacts on genome evolution (e.g., gene duplication and loss, other genome rearrangement events, etc.). As more divergent samples are included in a GWA study, more evolutionary processes potentially will become relevant to AM analysis. We fully expect that more algorithmic development will need to be done in this case, particularly regarding the breakpoint inference stage of Coal-Miner.

We conclude with our thoughts on future work. As an alternative to the pipeline-based design of Coal-Miner, simultaneous inference of local coalescent histories and AM model parameters will avoid error propagation across different stages of a pipeline-based algorithm. Furthermore, viewed through the lens of evolution, genotype and phenotype are arguably two sides of the same coin. The same could be said of "intermediate-scale" characters (e.g., interactomic characters). A combination of the extended coalescent models and LMMs could be used to capture evolutionary relatedness of and functional dependence between heterogeneous biological characters across multiple scales of complexity and at higher evolutionary divergences.

\section{SUPPORTING ONLINE MATERIALS (SOM)}

SOM files are located at https://doi.org/10.6084/m9.figshare.5165470. v1. These materials include: (1) an appendix with supplementary text, tables, and figures, (2) source code for software used in this study, and (3) datasets analyzed in this study. All materials are provided under open and free licenses.

\section{ACKNOWLEDGMENTS}

The authors gratefully acknowledge the following support: National Science Foundation Grants No. CCF-1565719 and No. CCF-1714417 (to KJL), grants from the BEACON Center for the Study of Evolution in Action (NSF STC Cooperative Agreement DBI-093954) to KJL and GAB, and Michigan State University faculty startup funds (to $\mathrm{KJL}$, to GAB, and to PPE). The authors would also like to thank the anonymous referees for their valuable feedback and suggestions.

\section{REFERENCES}

[1] Richard Amasino. 2010. Seasonal and developmental timing of flowering. The Plant fournal 61, 6 (2010), 1001-1013.

[2] Richard M Amasino and Scott D Michaels. 2010. The timing of flowering. Plant Physiology 154, 2 (2010), 516-520.

[3] Yoav Benjamini and Yosef Hochberg. 1995. Controlling the false discovery rate: a practical and powerful approach to multiple testing. Fournal of the Royal Statistical Society Series B (Methodological) 57, 1 (1995), 289-300.

[4] Carlo E Bonferroni. 1936. Teoria statistica delle classi e calcolo delle probabilita. Libreria internazionale Seeber. 
[5] Richard P. Brent. 1973. Algorithms for Minimization without Derivatives. Dover Publications, Mineola, New York. 1-208 pages.

[6] Peter E Chen and B Jesse Shapiro. 2015. The advent of genome-wide association studies for bacteria. Current Opinion in Microbiology 25 (2015), 17-24.

[7] 1001 Genomes Consortium. 2016. 1,135 genomes reveal the global pattern of polymorphism in Arabidopsis thaliana. Cell 166, 2 (2016), 481-491.

[8] Elizabeth R DeLong, David M DeLong, and Daniel L Clarke-Pearson. 1988. Comparing the areas under two or more correlated receiver operating characteristic curves: a nonparametric approach. Biometrics 44, 3 (1988), 837-845.

[9] Eric Y. Durand, Nick Patterson, David Reich, and Montgomery Slatkin. 2011 Testing for ancient admixture between closely related populations. Molecular Biology and Evolution 28, 8 (2011), 2239-2252.

[10] Scott V Edwards. 2009. Is a new and general theory of molecular systematics emerging? Evolution 63, 1 (2009), 1-19.

[11] Gregory Ewing and Joachim Hermisson. 2010. MSMS: a coalescent simulation program including recombination, demographic structure and selection at a single locus. Bioinformatics 26, 16 (2010), 2064-2065.

[12] Hussein A Hejase and Kevin J Liu. 2016. Mapping the genomic architecture of adaptive traits with interspecific introgressive origin: a coalescent-based approach. BMC Genomics 17, 1 (2016), 41.

[13] Richard R. Hudson. 2002. Generating samples under a Wright-Fisher neutral model of genetic variation. Bioinformatics 18, 2 (2002), 337-338.

[14] Richard R Hudson and Norman L Kaplan. 1985. Statistical properties of the number of recombination events in the history of a sample of DNA sequences. Genetics 111, 1 (1985), 147-164.

[15] Heqiang Huo, Shouhui Wei, and Kent J. Bradford. 2016. DELAY OF GERMINATION1 (DOG1) regulates both seed dormancy and flowering time through microRNA pathways. Proceedings of the National Academy of Sciences 113, 15 (2016), E2199-E2206.

[16] Ian Jolliffe. 2002. Principal Component Analysis. Wiley Online Library.

[17] Hyun Min Kang, Jae Hoon Sul, Susan K. Service, Noah A. Zaitlen, Sit-yee Kong, Nelson B. Freimer, Chiara Sabatti, and Eleazar Eskin. 2010. Variance component model to account for sample structure in genome-wide association studies. Nature Genetics 42, 4 (Apr 2010), 348-354.

[18] John F. C. Kingman. 1982. On the genealogy of large populations. Fournal of Applied Probability 19 (1982), pp. 27-43.

[19] Kevin J. Liu, Jingxuan Dai, Kathy Truong, Ying Song, Michael H. Kohn, and Luay Nakhleh. 2014. An HMM-based comparative genomic framework for detecting introgression in eukaryotes. PLoS Computational Biology 10, 6 (06 2014), e1003649.
[20] Anthony D Long and Charles H Langley. 1999. The power of association studies to detect the contribution of candidate genetic loci to variation in complex traits. Genome Research 9, 8 (1999), 720-731.

[21] Dustin Mayfield, Z Jeffrey Chen, and J Chris Pires. 2011. Epigenetic regulation of flowering time in polyploids. Current Opinion in Plant Biology 14, 2 (2011), $174-178$.

[22] Belén Méndez-Vigo, F. Xavier Picó, Mercedes Ramiro, José M. Martínez-Zapater, and Carlos Alonso-Blanco. 2011. Altitudinal and climatic adaptation is mediated by flowering traits and FRI, FLC, and PHYC genes in Arabidopsis. Plant Physiology 157, 4 (12 2011), 1942-1955.

[23] J. Chris Pires, Jianwei Zhao, M. Eric Schranz, Enrique J. Leon, Pablo A. Quijada, Lewis N. Lukens, and Thomas C. Osborn. 2004. Flowering time divergence and genomic rearrangements in resynthesized Brassica polyploids (Brassicaceae). Biological Journal of the Linnean Society 82, 4 (2004), 675-688.

[24] Stephanie S Porter, Peter L Chang, Christopher A Conow, Joseph P Dunham, and Maren L Friesen. 2017. Association mapping reveals novel serpentine adaptation gene clusters in a population of symbiotic Mesorhizobium. The ISME fournal 11, 1 (2017), 248-262.

[25] Sergej Potapov, Werner Adler, Benjamin Hofner, and Berthold Lausen. 2013. Daim: Diagnostic accuracy of classification models. R package version 1.1.0.

[26] Alkes L Price, Nick J Patterson, Robert M Plenge, Michael E Weinblatt, Nancy A Shadick, and David Reich. 2006. Principal components analysis corrects for stratification in genome-wide association studies. Nature Genetics 38, 8 (2006), 904-909.

[27] R Core Team. 2015. R: A Language and Environment for Statistical Computing. R Foundation for Statistical Computing, Vienna, Austria.

[28] Ying Song, Stefan Endepols, Nicole Klemann, Dania Richter, Franz-Rainer Matuschka, Ching-Hua Shih, Michael W. Nachman, and Michael H. Kohn. 2011. Adaptive introgression of anticoagulant rodent poison resistance by hybridization between old world mice. Current Biology 21, 15 (2011), 1296 - 1301.

[29] Jeremy Wang, Kyle J. Moore, Qi Zhang, Fernando Pardo-Manual de Villena, Wei Wang, and Leonard McMillan. 2010. Genome-wide compatible SNP intervals and their properties. In Proceedings of the First ACM International Conference on Bioinformatics and Computational Biology (BCB '10). ACM, New York, NY, USA, $43-52$.

[30] Oscar Westesson and Ian Holmes. 2009. Accurate detection of recombinant breakpoints in whole-genome alignments. PLoS Computational Biology 5, 3 (03 2009), e1000318.

[31] Xiang Zhou and Matthew Stephens. 2012. Genome-wide efficient mixed-model analysis for association studies. Nature Genetics 44, 7 (2012), 821-824. 